\title{
ACERCAMIENTO DEL SER ANCIANO AL SER ENFERMERO
}

\author{
Carmen Chamizo Vega \\ Enfermera e Historiadora de la Enfermería \\ Cl. Dindurra, 8-3 $3^{\circ}$ B - 33201 Gijón (Asturias) \\ Telf.: 985344359 • Fax: 985390753
}

\section{RESUMEN}

Este artículo pretende la aproximación de dos colectivos sociales históricamente marginados, como es el de los ancianos y es el de las enfermeras. Dado que, en la actualidad, el grado de relación que se produce entre ambos ha adquirido el grado de relación laboral, se aboga por una actitud de respeto y amor con el fin último de prestar unos cuidados profesionales y de calidad. De ahí que, conocer a la persona a cuidar, consensuar un modo de acción, dialogar e intercambiar conocimientos y experiencias, sean acciones inexcusables a la hora de cuidar.

\section{CLOSENESS OF THE ELDERLY TOWARDS THE NURSE}

\section{SUMMARY}

The present study explores the closeness between two different groups who have been historically marginated: the elderly and the nurses.

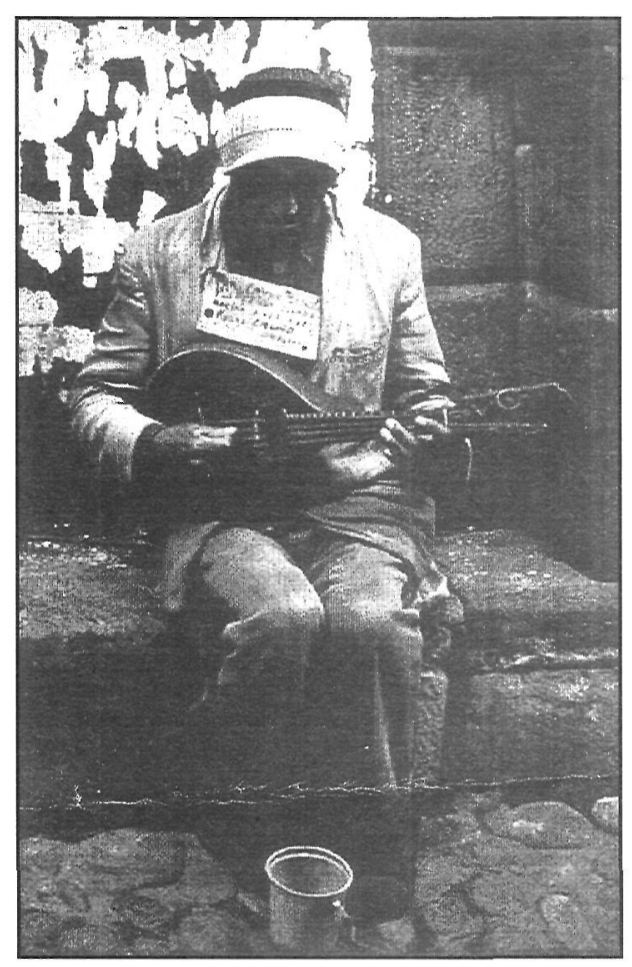

Due to the fact that today both groups are related by professional links this study concludes that an attitude of respect and a sort of friendship should be established between them in order to obtain a high professional and quality care.

To be able to meet this aim, dialogue, exchange of experiences and mutual help should be increased.

\section{[NTRODUCCIÓN}

Con este artículo se pretende llamar la atención ;obre la relación de " acercamiento " que ambos anciano/a y enfermero/a) experimentan a través del acto de cuidar. El primer paso realizado antes lel desarrollo del tema, fue verificar el significado te las palabras:

ICERCAMIENTO: acción de acercar, "poner a menor distancia "
DEL: contracción (se utiliza "del", en ambos casos, para dar sensación de dinamismo e interrelación y no "del ser anciano al ser enfermero ")

SER: existir, naturaleza, "esencia "

ANCIANO: persona de mucha edad

ENFERMERA/O: persona que asiste a los enfermos

ASISTIR: hallarse cerca, CUIDAR dar cuidado

CUIDADO: solicitud, atención para hacer bien una cosa.

Del latín cogitatum - que presta atención. 
Tras observar la concordancia de las definiciones con lo que se quería expresar y, tras realizar la búsqueda bibliográfica pertinente, comenzaron las reflexiones y anotaciones : ¿ qué es ser anciano, para un anciano ? y ¿que es ser enfermera, para las enfermeras /os ?... Estas preguntas se realizaron en la consulta, los hogares y los centros de trabajo.

La colección de respuestas a la primera pregunta(¿que es para usted ser ser anciano ?) fue variopinta y diversa : "ser una carga","tener mucha experiencia ", "haber trabajado mucho ", " no servir para nada ", "tener muchos dolores", " ser tolerante", "morise poco a poco ", "tener capacidad de dar", "ser un maniático ", "haber sufrido mucho "... etc. Conscientes de que muchos ancianos actuales vivieron la postguerra española, los que pasaron hambre, sufrimientos, trabajo duro, vieron morir a muchos, bailaban con gaita y tambor y no se criaron con la televisión, ni en una sociedad consumista, pero vieron un sistema de jubilaciones y un nivel adquisitivo mejor. Todo ello no coincidía con lo que respondía y menos aún, coincidía con la visión social de los ancianos: por su capacidad de escucha por su experiencia, por su tiempo de ocio bien utilizado, por su tolerancia por su capacidad de sufrir en silencio, etc. ; con la realidad que algunos ancianos intolerantes, egoistas y amargados mostraban.

Pero antes de continuar adelante, se propone al lector pensar sobre ¿qué entienden por "anciano"?

La respuesta dependerá del conocimiento del concepto, que socialmente esté reconocido y de las vivencias que se tengan. Pero, ahora bien, si se les pregunta ¿cómo se ven ustedes de anciano?" (intenten proyectarse en el futuro durante unos segundos) responderán " no me imagino ", "igual que ahora, pero con canas, arrugas, con un bastón, cargados de nietos, sin trabajar,..... es decir, el eje central de ese anciano serían ustedes mismos, los de ahora, pero con connotaciones y adjetivos generalmente externos, que nos hacen proyectar lo que caracteriza la última etapa de la vida, para cada uno de nosotros.

Otro tanto ocurriría si preguntara, “¿Qué es ser enfermera /o? ". Según el conocimiento o vivencias del entrevistado, las preguntas son tan dispares como numerosas: " una vocación, la / el ayudante del médico, la / el que hace curas y pone inyecciones, la / el que atiende las necesidades de los individuos, el / la que acompaña, etc.... Pero si la pregunta se concreta a los profesionales y es : "¿ Que es ser enfermera /o para vd. que es enfermera /o ? ", la respuesta reflejaría aquello que ella / él es y hace como enfermera /o.

Todo ello nos lleva a pensar que cada anciano y enfermera tiene una manera de ser. Esa manera de ser es lo que se llama programa vital o "yo" de cada uno, el cual elije entre diversas posibilidades de ser que en cada instante se abren ante él. Por ejemplo, ustedes podrían escoger entre una revista u otra, entre seguir leyendo estas líneas o no (siempre teniendo en cuenta las circustancias y la libertad) Pero estas decisiones no se podrían tomar si no se tuvieran convicciones o creencias sobre lo que sor las cosas, los hombres, nosotros mismos. Tomandc el ejemplo anterior, si ustedes se deciden a segui] leyendo esta revista o artículo, será porque estar convencidos de hacerlo,.. porque les interesan estos temas,... porque están sentados cómodamente y he oído hablar de ella,.. o puede ser que no tengan otra cosa mejor que hacer.

Así pues, las creencias no son ideas que se ra zonen, sino que se creen. Por eso se dice que er ellas el hombre está, ES. El creer que ser anciano de una manera u otra, o enfermera /o de un tipo u otro no depende del intelecto, sino de una manera de vi vir, algo que nos orienta para actuar de una maner: u otra, tanto personal como profesionalmente.

Podríamos decir pues, en un intento - exagerad quizás - de sintetizar, que la creencia actual es qui el anciano es un problema en sí (tanto social, sani tario, familiar, etc.) y que la enfermera (aunque es tá cambiando) es la que realiza curas corporales I aplica los tratamientos médicos.

Ahora bien, si preguntamos a los ancianos y : las enfermeras, si coincide la creencia personal col la expuesta anteriormente, las respuestas de nuevo serían muy diversas y hasta pudiera ser contrarias ¿Qué se pretende con esto? Que nuestra creenci; personal, podrá ser contraria a la opinión o creenci: social, pero no sustrae quilate alguno a la realidad

\section{DESARROLLO DEL TEMA}

Es pues necesario, revisar la historia y compara las creencias en los diferentes momentos y conse 
guir una comprensión de la vigencia o creencia colectiva actual, para contrastarla posteriormente con la personal. Referente a la ancianidad, se observa como en la época medieval y antes, envejecer era una CASUALIDAD. Durante el Renacimiento, el Barroco y la Revolución Científica pasó a ser un LUJO, debido a las epidemias. Ya durante el siglo XIX y XX comienza a ser CUESTIÓN DE TIEMPO y actualmente se contempla como un PROBLEMA, ya que las personas de edad avanzada representan una parte cada vez mayor de la población total y este fenómeno, está acompañado por la creciente preocupación de cómo cuidar a los ancianos, siendo cada vez mayor la cantidad de hogares para ancianos y los sistemas de jubilación, indicio de tal preocupación. Abordemos ahora, las creencias que sobre la profesión enfermera existiron y para ello sirva un breve repaso a nuestro pasado, según palabras de Françoise Colliére. La mujer enfermera era la que cuidaba o prestaba atención y se hallaba cerca, cuando lá necesitaban - Mujer cuidadora -. Acompaña al tiempo y aumenta el concepto religioso en el cuidado - Enfermera Religiosa - y posteriormente el concepto de razón y ciencia la supedita al conocimiento científico - médico - Enfermera dependiente de la Institución -. El empuje del Feminismo en los años 70, hace que comience a ponerse en tela de juicio, el control que los médicos ejercían sobre la profesión enfermera y se accede a los niveles de decisión caracterizados como masculinos, surgiendo contradicciones al desarrollar el aspecto femenino de los cuidados que se realizan. Actualmente, los cuidados enfermeros se han convertido en un analizador socio - económico - político, pero los cuidados no son el objetivo de las instituciones sanitarias, sino el tratamiento de la enfermedad.

Se puede concluir entonces que, tanto la opinión vigente sobre el anciano y sobre la Enfermería, no es la opinión social deseada; por lo que se cree que ancianos/as y enfermeros/as - quizás otros también - deberían ser activos individualmente y sentirse protagonistas e interlocutores en un mundo social y laboral que hasta ahora se aprovechó de la sumisión de ambos.

Hasta aquí, se ha intentado buscar el fondo y los rasgos generales de los sujetos: el anciano/a y la en- fermera /o. Pero, cuando se intenta fotografiar una caricia, una gota de rocío resbalando por un pétalo, o un insecto libando el néctar de una flor, es porque se intenta perpetuar ese momento, ese sentimiento, la luz, la armonía que se desprende de esa relación. Es por ello, que se hizo hincapié en la profundización de ciertos valores elementales y necesarios para lograr la armonía de los cuidados en los ancianos/as. Es claro que el nivel ético de las realizaciones humanas siempre ha sido bajo; mientras que el nivel de las realizaciones técnicas se ha elevado rápidamente, estando hoy más que nunca, muy alejadas la ética y la técnica. Pues bien, LA DIGNIDAD HUMANA - ese valor común por la vida que todos reconocemos -, sólo puede alcanzarse en el terreno de la ética; y los logros éticos se miden por el grado en que los actos estén gobernados por LA COMPASIÓN (sentimiento doloroso ante la desgracia ajena) EL AMOR sentimiento afectivo que busca el bien y desea poseerlo) y LA TERNURA (esmero con que se hace una cosa) y no por LA CODICIA (deseo inmoderado de riquezas u otras cosas) y LA AGRESIVIDAD (actitud violenta de atacar).

Deténganse por un momento amigos lectores i por qué están presididos los cuidados a los ancianos ?. Se puede decir que están presididos por la prisa, por el exceso de técnica, de trabajo, de conocimientos teóricos, por "encorsetados" protocolos, por el hacer sin tener tiempo a reflexionar, por el cansancio personal,etc... Todo ello, conduce a un sentimiento de impotencia, que se traduce en una agresividad " hacia afuera " (la culpa es de....) y no de una actitud positiva y reflexiva (¿Que ocurre?, ¿Por que me afecta?, ¿Qué siento ?) con lo que el valor de la DIGNIDAD HUMANA (la del personal de enfermería y la de los que cuidamos) va decayendo, llegando incluso al punto de sentir apuro al utilizar términos o sentimientos de compasión y amor (sentimientos de dignidad, al fin y al cabo) que si hacemos memoria, estan presentes cuando se elige la Enfermería como profesión.

En lenguaje práctico, es hora de dejar el campo de la enfermedad y pasemos al campo y origen de los cuidados : LA VIDA en la que se incluye la enfermedad y la muerte). Sustituyamos preguntas como ¿cúantos ancianos tenemos? ¿cúantos son diabéticos, hipertensos, seniles, encamados? ¿qué pro- 
tocolo de cuidados utilizaremos?, por otras que aporten conocimientos sobre aquello que ayuda a vivir (o a morir) a los ancianos. Serán los ancianos los que nos digan hasta dónde sufren, saben y quieren que hagan las enfermeras ; y serán las enfermeras las que les ayuden a valorar la vida, la muerte y los diferentes conocimientos que tengan. En resumen y para finalizar, se hace énfasis en dos objetivos comunes (ancianos/as y enfermeras/os) : Los cuidados para la vida y/o la muerte y los conocimientos personales de ambos. Adecuando ambos objetivos a una relación de feed-back permanente, se logra (anciano/a - enfermera/o) una satisfacción personal y profesional, consiguiendo así, la armonía de la que antes escribía.

\section{SITUACIONES COMUNES EN LOS ANCIANOS : A MODO DE REFLEXIÓN.}

Aunque se parte siempre de la situación individual de cada anciano/a y de cada enfermera/o, se comentan algunos de los momentos comunes por los que pasan los ancianos y que son puntos de reflexión y partida de los cuidados enfermeros.

\section{- LA PÉRdIDA DE TRABAJO REMUNE-} RADO (más frecuentemente en el hombre actualmente) y en algunas mujeres el relegarles de las tareas del hogar ("antes hacía la comida...,organizaba la casa...). Generalmente existe una alteración de la Autoimagen, y de la necesidad de pertenencia, de espacio,etc... Es una fase de adaptación al nuevo papel, debiendo estar nuestros cuidados encaminados a facilitar apoyo individual, fomentar grupos de auto-ayuda y a cambiar estereotipos sociales ( $\mathrm{Ya}$ está jubilado! ¡no vale para nada!.). Como consecuencia existe :

\section{- UN AUMENTO DEL TIEMPO LIBRE O} DE OCIO que si no se ha sabido ocupar previamente, da lugar a un "vacío". Aparecen las dependencias (de los hijos, de los nietos, de los medicamentos, del psicólogo..). Unos cuidados eficaces irían dirigidos a la prevención, fomentando la ocupación del tiempo libre y satisfactorio en la madured.

- APARECEN LAS INCAPACIDADES Y LAS ENFERMEDADES. Es aquí más desarrolla- da está la Enfermería por lo que no nos extenderemos más. Sólo decir que es importante observar lo que experimenta el anciano/a ante las incapacidades.

- LOS TEMORES: En este apartado se incluye toda una miscelanea de preocupaciones que abruma a los ancianos y que desemboca en un aumento de angustia y ansiedad. Un tema que les preocupa enormemente es LA MUERTE, la de los más cercanos (esposo/a, amigoas/os..) e incluso la suya propia. Otro sentimiento negativo que perciben es la SOLEDAD, pero la soledad del que está solo no es la peor, porque aún le queda la esperanza ; pero la soledad del que está acompañado por quien no le corresponde, sólo le queda la desesperación. i Quién no ha visto esta soledad en algunas familias o casas donde hay ancianos?. Todo anciano es una persona con un vagaje de la vida y la importancia y significación de cómo afronte la soledad, no reside en la compañía externa sólo, sino en la capacidad de la persona en " ahuecarse " (como un botijo) hasta el punto de que pudiera caber y entrar libremente, en ella, diversas situaciones siendo así dueña de las mismas, e incluyendo la soledad, que al fín y al cabo, el más y mejor acompañado, tendrá que afrontar tarde o temprano : la soledad de La Muerte. ¿Cómo se consigue esto ? Según las enseñanzas de algunos ancianos, ejerciendo a lo largo de la vida La Tolerancia. Y ¿ qué pueden hacer las enfermeras ? : Ser, estar, acompañar auténticamente a través de los cuidados, es nuestra respuesta.

Otro aspecto que le hace sentir angustia al anciano/a, es el concepto del TIEMPO, muy diferent $\epsilon$ al concepto del tiempo que poseen sus cuidadores. Para ilustrar el concepto de TIEMPO valga una frase que Michel Ende escribió en el libro de Momo : "Nadie se daba cuenta que al ahorrar tiempo, ahorraba otra cosa, su vida se volvía cada vez más pobre, más monótona, más fría. Los que lo sentían con claridad eran los niños y los ancianos, pues nadie tenía tiempo para ellos. No se daban cuenta que e] tiempo es Vida y la vida reside en el corazón “.

Cuanto más se trabaja, más se gana, menos se vive y menos tiempo se tiene para recrearte en la belleza, en la contemplación, en el desarrollo de los 
sentidos - qué sienten, a qué huelen, que tacto tienen, qué agradecen, qué ven... los ancianos -.

El anciano/a no tiene prisa, camina hacia atrás, mirando hacia la vida pasada y dirigiéndose hacia la muerte, como Momo caminaba hacia la Casa de Ninguna Parte.

En dicha obra se lee también: "El que a uno le gustara su trabajo y lo hiciera con amor, no importaba ; al contrario eso sólo entretenía. Lo único importante era que hiciera el máximo trabajo en el mínimo de tiempo ". Esto hace refelxionar en la mala adecuación del tiempo en el trabajo como enfermera y tal como los ancianos lo necesitan. Es un punto que se debe debatir ampliamente entre el personal de enfermería, para dar alternativas a las Instituciones, la Comunidad, la familia.. etc.

La reflexión de todo lo anteriormente expuesto sería que las/los enfermeras/os debemos estar atentos y observar aquello que los ancianos dicen, hacen, viven, en suma aquello que SON Y QUIEREN SER, para que cuando pasen por uno o varios momentos difíciles, les prestemos atención atenuando sus temores y estando cerca de ellos. Esto sólo se hace estando con ellos, trabajando con los ancianos /as, descubriendo y aprendiendo con sus problemas y necesidades. No se hace sentado en los despachos, no se obtiene con títulos solamente, no se conoce por estadísticas ; esto nos puede ayudar a comprenderles mejor, pero ningún tratado de conocimientos puede suplir la cuidadosa valoracón que cada uno de ustedes hace cuandoacude a casa de
Manuel o atiende a María y sabe cómo vive, vivió, cúal es su historia de vida, sus creencias, su entorno, su familia, su enfermedad si la tuviera, la aceptación de sí mismo que tiene, el olor de sus úlceras, etc.... Es a partir de entonces, cuando tendremos un conocimiento del anciano, que sumado a los conocimientos de vida personales y profesionales, nos facilitaran la tarea de elaborar de forma eficaz y real los Cuidados enfermeros y no de manera ideológica, como pudiera creerse. Esta, creo que es la mayor aportación que podemos, como enfermeras/os, hacer a los ancianos, a la comunidad, al equipo multidisciplinar y en suma a la sociedad.

\section{BIBLIOGRAFÍA}

Bender, M. \& Lloyd, C. (1996) Calidad de muerte: Atención al anciano en la etapa final de su vida. Tea, Madrid.

Colliere, M. F. (1993) Promover la vida. InteramericanaMcGraw Hill, Madrid.

Martínez Fornes, S. Y Fermín Gómez, F. (1991) Envejecer en el año 2000. Instituto Nacional de Servicios Sociales, Madrid.

Orts Montenegro, A. \& Laguna, A. (1997) Reflexiones sobre el envejecimiento y la calidad de vida. Cultura de los Cuidados 2, 60-63

Pérez Melero, A. (1995) Enfermería Geriátrica. Síntesis, Madrid.

\section{$\Delta$

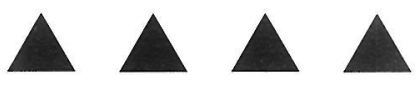 \\ $\Delta \Delta \Delta \Delta \Delta \Delta \mathbf{A}$

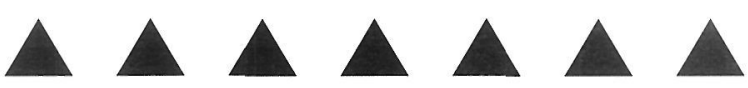

